\title{
La alomorfía en el plural fracto árabe. Del Kitāb de Sībawayhi al árabe moderno. El caso del esquema singular $f a^{\prime}$ ' $a l$
}

\author{
Allomorphy in the Arabic broken plural. \\ From the Kitāb by Sībawayhi to Modern Arabic. \\ The case of the singular pattern $f a$ ' $a l$
}

\author{
Ignacio Ferrando \\ Universidad de Cádiz \\ http://orcid.org/0000-0002-7335-4243
}

\begin{abstract}
Ofrecemos en este trabajo un estudio de contraste entre la descripción que hace Sībawayhi, el más reconocido de los gramáticos árabes, de los esquemas de plural fracto de uno de los esquemas de singular, fa 'al (a modo de ejemplo) y los datos extraídos de algunas obras lexicográficas árabes y del corpus de textos árabes reunidos en algunas bases de datos disponibles. Una vez analizado el método del gramático de Basora en la recogida y sistematización de datos, el objetivo es comprobar si la información que proporciona coincide con la aportada por los diccionarios y textos árabes. Estudiamos de forma particular el tratamiento de los casos de alomorfía, esto es, el uso de varios plurales para un mismo singular, tratando de dilucidar si se trata de una alomorfía libre o motivada.
\end{abstract}

Palabras clave: plural fracto; Sībawayhi; gramática árabe; morfología árabe; historia de la lengua árabe
We present in this paper a contrastive study of the broken plural patterns taken by the singular pattern fa 'al (by way of example), according to the information provided by Sībawayhi, the most renown of Arab grammarians. His data are confronted with the information gathered in some Arab lexicographical works and a corpus of Arab texts in some available databases. After analysing the method of the Basra grammarian in his collection and systematization of data, the goal is to check if the information collected by him matches the information provided by Arabic dictionaries and texts. We study in particular the discussion of allomorphy cases, that is to say, the use of several plural patterns for a given singular, trying to elucidate whether this kind of allomorphy is free or conditioned.

Key words: Broken plural; Sībawayhi; Arabic grammar; Arabic morphology; History of Arabic.

El punto de partida de este trabajo es el choque lógico que se produce en la mente de cualquier estudiante occidental de la lengua árabe cuando descubre la riqueza y perfiles tan diversos de la categoría de

Copyright: (C) 2018 CSIC. Este es un artículo de acceso abierto distribuido bajo los términos de la licencia de uso y distribución Creative Commons Reconocimiento 4.0 Internacional (CC BY 4.0). 
número en esa lengua semítica (en menor medida en otras lenguas semíticas como el etíope o el sudarábigo, mucho menos en el hebreo y el arameo $\left.^{1}\right)$. Y es que una persona acostumbrada a considerar el número como una simple oposición cuantitativa entre singular (1) y plural $(1+$ $\mathrm{x})$, se encuentra en una situación difícil para asimilar la concepción de la pluralidad en árabe ${ }^{2}$, que incluye, desde el punto de vista meramente cuantitativo, no solo un número dual $(1+1)$, sino también un plural de pequeño número o paucal $(1+2-9)$ y un plural de gran número o multitudinal $(1+10-\ldots)$. A esto se le suma, desde el punto de vista cualitativo, la existencia de un colectivo que funciona como una suerte de plural grupal frente a otro tipo de plural de carácter más individualizado $^{3}$. Pero no solo esto, sino que desde el punto de vista morfológico el árabe dispone de dos mecanismos completamente diferentes para formar los plurales: la sufijación, similar a la de las lenguas neolatinas, con morfemas diferentes para masculino y femenino, y el llamado "plural fracto" o plural interno, consistente en la modificación de la estructura del singular, incluyendo infijación, reduplicación consonántica, y adición, supresión, modificación, alargamiento o acortamiento de vocales $^{4}$. Y aún hay más, y quizá esto es lo que más sorprende a quien se acerca al estudio de esta lengua: el uso de diferentes formas de plural para un mismo singular, algo bastante habitual en todos los periodos de la lengua árabe y que desconcierta a quien trata de aprenderla. No

\footnotetext{
${ }^{1}$ Mucho se ha debatido la cuestión del origen del plural fracto. Algunos semitistas defienden que se trata de una innovación del semítico meridional, mientras que otros lo consideran un rasgo protosemítico, incluso protoafroasiático, prácticamente desaparecido en el semítico septentrional. Si bien nos inclinamos por la primera propuesta, no es este el lugar para entrar a fondo en ello. Véase la opinión de Ratcliffe (The broken plural problem in Arabic and comparative Semitic, pp. 150-152), favorable a la primera propuesta, la de Corriente (Problemática de la pluralidad en semítico, pp. 74-75 y 1996:38-39), partidario con matices de la primera, y las visiones de Hetzron ("Two Principles of Genetic Classification", p. 102) y Zaborski ("How Conservative and How Innovating is Arabic?", p. 44), defensores de la segunda.

${ }^{2}$ Situación descrita de forma excelente y sucinta en Corriente (Problemática de la pluralidad en semítico, p. 5).

${ }^{3}$ Véanse más detalles sobre este tipo de plural "individualizador" en Ferrando, "Plurals of Paucity in Arabic. From Ancient to Modern Usage" y Ferrando, "Al-muțābaqa bayna n-na 'ti wa-l-man 'ūti fì riwāyāti Yūsuf Zīdan".

${ }^{4}$ Es notable que dos de las monografías más brillantes escritas sobre el plural fracto incluyen la palabra "problema" en el mismo título. Nos referimos a Corriente, Problemática de la pluralidad en semítico y Ratcliffe, The broken plural problem in Arabic and comparative Semitic.
} 
es en absoluto infrecuente toparse con dos o tres plurales para un mismo singular, en ocasiones con perfiles semánticos diferentes, en ocasiones en (aparente) libre alternancia. Y hay incluso algunos (raros) casos de singulares que disponen de hasta ocho plurales diferentes. Este fenómeno de alomorfía ${ }^{5}$ ha invitado desde antiguo a los investigadores a tratar de desentrañar los motivos y orígenes de tal situación de "hipertrofia", contraria en principio a los principios de economía lingüística más comunes.

De este choque lógico surge, inevitablemente, una serie de preguntas que se hace el estudiante del árabe y que constituyen el germen de este estudio: ¿A qué responde esta riqueza lógica y morfológica del plural en árabe? ¿Se trata de los restos de un sistema primitivo de pluralidad, o de un desarrollo ulterior con alto grado de variación debida a las diversas modalidades diatópicas y diafásicas de la lengua árabe? ¿Cómo fue el desarrollo histórico del plural fracto? ¿Ha ido el hablante perdiendo gradualmente la consciencia de un sistema multipolar del plural? ¿Refleja la situación actual la evolución hacia patrones más sencillos, hacia lo meramente cuantitativo? ¿La alomorfía en el plural fracto se debe básicamente a la diversidad dialectal ${ }^{6}$, o hay otras razones de índole fonética o semántica? ¿Es simplemente un rasgo léxico, como se indica en muchas gramáticas y tratados de la lengua árabe, tanto occidentales como árabes? ¿Cómo han ido evolucionando los distintos esquemas de plural a través de las distintas fases históricas de la lengua árabe?

En segundo lugar, este trabajo se inspira en la lectura de la excelente y pionera obra de Corriente (1971), en la que, con un enfoque eminentemente diacrónico y de lingüística comparada, se pasa revista a los distintos perfiles del plural fracto en árabe y en otras lenguas semíticas. Se trata de una obra muy importante que arroja luz sobre el origen del sistema del plural fracto y sobre los perfiles morfológicos del mismo, y que nos sirvió, durante muchos años, de inspiración para diversas in-

\footnotetext{
${ }^{5}$ Véase sobre este concepto Lloret ("La alomorfía en la teoría de la optimidad", pp. 133-135).

${ }^{6}$ Así lo afirma as-Sāmarrā'1 (Fiqh al-luga al-muqāran, p. 123): “wa-kațratu șiyaġi ğumū 'i $t$-taksīri fì l- 'arabiyyati tastar' $\mathrm{l} t$-ta'ammula wa-n-nazara bi-haytu là nastațī $u$ an nufassira dâlika bi-ġayri l-qawli bi-ta 'addudi l-lahağât", es decir: "La abundancia de esquemas de plural fracto en árabe invita a reflexión y examen, de forma que no podemos explicarla sin hablar de la diversidad dialectal".
} 
vestigaciones en torno al plural árabe. Es digna de encomio la altura de miras de F. Corriente en este trabajo, puesto que no se limita a presentar los hechos desde un punto de vista meramente sincrónico y "árabe", sino que amplía el espectro a la diacronía y a la semitística comparada. Sin embargo, únicamente se ocupa de forma pasajera de una de las cuestiones que más nos interesan aquí, esto es, de la variación alomórfica detectada en los plurales fractos del árabe, e incluso la variación moderna entre plural fracto y plural regular para un mismo singular, que viene a demostrar la productividad de un viejo fenómeno, el de la presencia / creación de dos plurales para un mismo singular, en este caso con posible discriminación semántica entre ambos ${ }^{7}$.

En tercer lugar, nuestra investigación toma realmente cuerpo a partir de un descubrimiento feliz: la lectura de las páginas 567-650 del tercer volumen del Kitāa de Sībawayhi, el célebre gramático de Basora ${ }^{8}$. En esas 84 páginas se diseccionan con precisión y brillantez los esquemas del plural fracto, poniendo siempre el énfasis en la variación alomórfica que tanto nos llama la atención. Sībawayhi parte siempre del esquema del singular, y ofrece para todos y cada uno de los esquemas en primer lugar la forma de plural de pequeño número (paucal), y en segundo lugar la forma de gran número (multitudinal). Tras ello comienza a tratar las excepciones a la norma y las formas que no se ciñen a lo más habitual. A menudo el genial lingüista de Basora ofrece informaciones sobre la frecuencia de unos u otros esquemas, o sobre la adscripción geográfica o tribal de algunos otros, aunque no de forma plenamente sistemática. También presta una gran atención a los perfiles fonéticos del singular a la hora de la construcción del esquema plural. Por ejemplo, trata de forma diferenciada los singulares con reduplicación de la segunda consonante radical, así como los singulares cuya segunda consonante es una "defectiva" $(y / w)$. La descripción que ofrece es, en sí misma, un análisis exhaustivo del plural fracto que pone de relieve la

${ }^{7}$ Véase un análisis de este fenómeno desde el punto de vista del uso real en la lengua moderna en Ferrando Frutos, "Broken versus regular plural in Modern Arabic: the case for taf 'il", aplicado a un esquema concreto, el mașdar del verbo derivado en forma II, es decir, $t a f^{\prime} i ̄ l$, que admite dos plurales diferentes: taf'īlāt (regular, por medio de sufijación) y tafā îl (fracto).

${ }^{8}$ En la edición de Hārūn (Kitāb Sībawayhi). Véase nuestro trabajo "Sībawayhi y el concepto de jam 'u l-qilla", en el que analizamos en detalle la información aportada por Sībawayhi. 
riqueza de este rasgo morfológico-semántico ${ }^{9}$. En nuestra opinión, esta riquísima y detallada descripción no ha sido apenas tenida en cuenta por los investigadores occidentales que han estudiado la cuestión del plural fracto, puesto que se han centrado en aspectos cuantitativos o de análisis morfológico (como Ratcliffe 1998), o en aspectos estadísticos, diacrónicos y comparados (como Murtonen 1964 y Corriente 1971), pero no han estudiado a fondo la variación alomórfica a partir de los datos ofrecidos por Sībawayhi y de los testimonios escritos de la lengua árabe en sus diferentes épocas.

Con esa idea en mente, la de investigar la variación presente en los esquemas del plural fracto en todas las fases históricas de la lengua árabe, hemos publicado en las dos últimas décadas una serie de trabajos de investigación dedicados, desde diversos ángulos y ofreciendo diferentes aproximaciones, al análisis de esta cuestión. En el primero de ellos, Ferrando (1999), partimos de la lectura y análisis del encomiable trabajo de Ratcliffe (1998) para plantear una suerte de state of the art de la cuestión del plural fracto en árabe y en semítico y hacer algunas observaciones sobre la variedad alomórfica del sistema. En el segundo de ellos, Ferrando (2001b), hicimos un análisis detallado del llamado paucal o plural de pequeño número (para entre 3-10 numerados) a partir de la abundantísima información proporcionada por Sībawayhi. Hay que señalar que este tipo de plural es quizá, al menos en la fase antigua de la lengua, el responsable de buena parte de la duplicidad de formas de plural para un mismo singular, aun cuando su uso haya ido decayendo con el correr del tiempo y haya sido incluso despreciado por lingüistas modernos ${ }^{10}$. En el tercer trabajo, Ferrando (2006), se presenta un estudio estadístico elaborado a partir de la poesía preislámica (las siete $m u^{c} a l l a q a \bar{a} t$ ) para comprobar si la categoría de paucal es productiva en esa fase y registro de la lengua árabe. La conclusión, aunque provisional, es bastante clara: los poetas preislá-

\footnotetext{
${ }^{9}$ Téngase en cuenta que Sībawayhi, tal como nos aclara de forma brillante Aryeh Levin en varios de sus trabajos (por ejemplo Levin "Sībawayhi's attitude to the language of the Quran"), o Baalbaki ("The Book in the grammatical tradition: development in content and method"), tiene como primer objetivo analizar y preservar el legado lingüístico de los árabes, y las fuentes en las que se basa, por orden de importancia, son: 1. La información oral de los beduinos; 2. El Corán; 3. La poesía preislámica y de los albores del Islam.

${ }^{10}$ Así hace, por ejemplo, Ḍayf (Taysīrāt lugawiyya, pp. 56-64), que propone eliminar la distinción entre paucal y multitudinal de las gramáticas descriptivas y normativas de la lengua árabe.
} 
micos, en línea con lo que defiende Sībawayhi, tienden a optar de forma consciente por un esquema de plural de pequeño número para referirse a un grupo pequeño y por un esquema de gran número para un número grande, si bien esto es válido únciamente para algún esquema como $f a ' l$ y, además, hay ciertos "cruces" entre ambos esquemas. En ese mismo artículo se estudia también, a partir de una cita del gramático de Kufa al-Farrā' ${ }^{\prime 1}$, otro fenómeno relacionado con el plural de pequeño número: la concordancia de sustantivos referidos a seres inanimados con adjetivo, verbo o pronombre en plural para indicar la individualización o el número pequeño (talātatu layālin halawna), frente a la concordancia de esos mismos sustantivos con un adjetivo, verbo o pronombre en femenino singular para indicar número grande o colectivización (țalātatu layālin halat). En el cuarto trabajo, Ferrando (2006-2007), se hace un análisis estadístico, a partir de corpus modernos, de la pervivencia de la distinción entre plural de pequeño y de gran número en algunos sustantivos en árabe moderno. En el quinto trabajo, Ferrando (2010a), se incide en la importancia de las páginas dedicadas por Sībawayhi al plural fracto en general y al de pequeño número en particular, y se subraya el hecho de que los gramáticos y lingüistas posteriores han tendido a pasar por alto esa rica información, simplificando en gran medida la aproximación a las diversas formas de plural. En el sexto trabajo, Ferrando (2010b), se ofrece un estudio estadístico, a partir de fuentes digitales disponibles en internet, de algunos sustantivos árabes de esquema tafīl (el mașdar de la forma derivada II del verbo trilítero) que reciben dos plurales diferentes, uno fracto $\left(t a f \bar{a}^{c} \bar{l} l\right)$ y otro regular con sufijo (taf $\left.\bar{\imath} l \bar{a} t\right)$, destacando que en varios de los casos más conspicuos cada una de las dos formas de plural adquiere un valor semántico diferente, lo cual viene a demostrar que el genio de la lengua tiene interiorizada la productividad semántica que encierra o puede encerrar la variación morfológica. En el séptimo y último trabajo, Ferrando [en prensa], se hace un análisis de la concordancia que se había tratado en Ferrando (2006) aplicado a las novelas del autor egipcio moderno Youssef Ziedan, en las que aparece con nitidez el uso de las dos concordancias posibles para diferenciar así dos tipos de plural: uno más "colectivizado" o grupal frente a otro más "individualizado" y particular.

${ }^{11}$ Véase Nağāầ y an-Nağğăr (Ma 'ānī l-Qur'ān li-abī Zakariyyāa al-Farrā', p. 453). 
Todos estos trabajos publicados sobre cuestiones relativas al plural fracto y al plural de pequeño número constituyen el terreno abonado para la propuesta de investigación que presentamos ahora, en la que trataremos de profundizar en este ámbito científico de trabajo, eligiendo para ello, a modo de botón de muestra, uno de los múltiples esquemas nominales árabes: $f a$ ' $a l^{12}$. El objeto último de la investigación será determinar las diferencias semánticas entre unas y otras formas del plural, si las hay, teniendo muy en cuenta el factor diacrónico, para lo cual haremos una labor constante de contraposición de datos antiguos frente a datos modernos. Para ello, vamos a cotejar los datos aportados por Sībawayhi con los datos lexicográficos presentes en un listado que hemos elaborado despojando los plurales del singular $\mathrm{fa}$ ' $\mathrm{al}$ en uno de los más célebres diccionarios clásicos, el Lisān al- 'arab de Ibn Manz̄ūr, dado su carácter exhaustivo, y en un diccionario bilingüe dedicado a la fase moderna de la lengua árabe, el de la edición inglesa de Hans Wehr. ${ }^{13}$ Dicho listado incluye un total de 539 singulares con esquema $\mathrm{fa}$ ' $\mathrm{al}$. Y también vamos a tratar de profundizar en el uso real de dichos plurales, para lo cual utilizaremos la base de datos ArabiCorpus, de Dilworth Parkinson ${ }^{14}$, que permite hacer búsquedas en un corpus muy amplio de textos árabes de fase antigua o de fase moderna, o bien en todo el corpus.

\section{Esquema $f a^{\prime} a l$}

Se trata de un esquema triconsonántico utilizado por la lengua árabe para diversas clases de sustantivos. Se usa con frecuencia para el mașdar del verbo fa 'ila yaf'al (hemos detectado 177 casos en nuestra lista), para sustantivos que designan un colectivo (49), para nombres de enfermedades y dolencias ( 21 casos) y para fitónimos y términos botáni-

${ }^{12}$ El motivo de la elección de este esquema y no otro es que se trata de uno de los que más variedad presentan en la formación del plural, solamente por detrás de $f a$ ' $l$, y al mismo tiempo testimonia cruces entre unos esquemas y otros que reflejan un cierto "bloqueo" de la discriminación entre los alomorfos disponibles para un mismo singular.

${ }^{13}$ Somos conscientes de que lo ideal sería manejar para esta tarea un diccionario árabe monolingüe. Pero, por desgracia, los diccionarios de este tipo tienden a recoger acríticamente los plurales incluidos en las obras más antiguas, pues no se basan en el corpus real de la lengua, sino meramente en la tradición lexicográfica.

${ }^{14}$ Disponible en formato libre y abierto en: arabycorpus.byu.edu. 
cos (19). En estos cuatro casos es muy poco frecuente que el singular fa 'al admita alguna forma de plural. Sí sucede en ocasiones que el mașdar, que en principio designa la acción verbal, y por lo tanto no admite plural, experimenta una cierta evolución semántica hacia sentidos más concretos y recibe un plural, generalmente de esquema $a f^{\prime} \bar{a} l$, como țarab "arrobo, emoción estética" pl. ațāb, țama "ambición" pl. atmā", "ăgab "sorpresa; admiración" pl. a ‘̆gāb, farah "alegría" pl. afrāh o wasah "suciedad" pl. awsāh. Los demás sustantivos con este esquema (273 en nuestra lista) designan seres u objetos concretos, y son por lo tanto susceptibles de recibir uno de los esquemas de plural fracto que analizamos a continuación.

Lo primero que señala Sĩbawayhi al tratar este esquema es que el plural de pequeño número, para referirse a un grupo de entre 3 y 10 seres u objetos, utiliza el esquema $a f^{\prime} ' \vec{l} l$, en tanto que el plural de gran

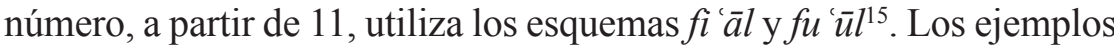
que cita son muy ilustrativos, puesto que dan pie a efectuar interesantes pesquisas sobre la productividad de la distinción entre ambas formas de plural, el paucal y el multitudinal. Son ğamal pl. ăgmāl y ğimāl, $\breve{g} a b a l$ pl. a ğb $\bar{a} l$ y ğibāl y asad pl. $\bar{a} s \bar{a} d$ y $u s \bar{u} d$. Y es que esos plurales de pequeño número de esquema af'a $l$ aparecen recogidos en los diccionarios, tanto en los diccionarios clásicos, del estilo del Lisān al'arab, como en los modernos, por ejemplo los de Wehr y Cortés, en este caso de forma un tanto acrítica y como por inercia, pero no se constata que sean formas vivas en la lengua árabe de hoy en día. De hecho, si hacemos una búsqueda en la sección denominada Modern Literature $^{16}$ en el ArabiCorpus de Dilworth Parkinson, no encontramos vestigio alguno de las tres formas de pequeño número ( $a \breve{g} m \bar{a} l$, a $\breve{g} b \bar{a} l$ y $\bar{a} s \bar{a} d$ ) frente a 13 casos de ğimāl, 43 de ğibāl y 98 de usūd. Además, el

${ }^{15}$ En cuanto a los perfiles morfo históricos de estas tres formas básicas para el plural del singular fa 'al, Corriente (Problemática de la pluralidad en semítico, pp. 10-12) propone que las dos formas $f i$ ' $\bar{a} l$ y $f u$ ' $u ̈ l$ pertenecen a un tipo morfológico con apofonía real caracterizado por la prolongación vocálica, e incluye la forma $a f^{\prime} a \bar{l}$ dentro de un subtipo del anterior, solo que con prefijo (aformativa), tan antiguo como los dos anteriores. Por su parte, Ratcliffe (The broken plural problem in Arabic and comparative Semitic, pp. 7781) propone que estas tres formas de plural son el resultado de añadir una vocal larga antes de la consonante final, solo que de distinto color ( $u$ vs. $a$ ), y que el aparente prefijo $a$ - de la forma $a f^{\prime} \bar{a} l$ podría ser el resultado, en realidad, de un proceso de metátesis.

${ }^{16}$ Sección compuesta por algo más de 1 millón de palabras, que comprende 32 novelas de diversos autores contemporáneos. Véase www.arabicorpus.byu.edu para más detalles. 
hecho de que los arabófonos apenas las conozcan, y que no sean capaces de identificar, para ninguna de estas voces, la diferencia entre un plural paucal y otro multitudinal ${ }^{17}$, nos indica que la evolución del idioma ha ido eliminándolas del acervo y que se ha truncado la discriminación que existía entre plural de pequeño y de gran número en esas voces, al no estar ya disponibles los esquemas de pequeño número en los textos modernos. El propio genio de Basora parece ser consciente de ello cuando señala lo siguiente: wa-fa' alun yuqtașaru fìhi 'alà adnà l- 'adadi katīira "en el esquema $f a$ 'al se usa de forma exclusiva el tipo de paucal con mucha frecuencia"18. Cabe preguntarse si el uso por parte de Sībawayhi de estas voces como los primeros ejemplos de su análisis del esquema $\mathrm{fa}$ ' $\mathrm{al}$ demuestra que se trataba, en el árabe que describe, de formas bien difundidas y conocidas. Aunque esto es algo difícil de contestar con precisión, puesto que, como ya se ha señalado, el bloque más importante de la información que ofrece el lingüista de Basora es oral y procede del árabe de los beduinos, sí se puede constatar que los textos de la sección que Parkinson etiqueta como Premodern ${ }^{19}$ recogen tímidamente esas formas de pequeño número (42 casos de ă̆măl, 27 de $a \breve{g} b \bar{a} l$ y 15 de $\bar{a} s \bar{a} d$, frente a 423 de ğimāl, 583 de $\breve{g} i b \bar{a} l$ y 325 de usūd). La conclusión parece clara: el plural de pequeño número, al menos para los sustantivos de esquema $\mathrm{fa} \mathrm{a}^{\prime} \mathrm{al}$, es un rasgo que no pertenece al árabe de fase moderna, y que en el árabe de fase antigua parece algo más bien residual. Al menos eso sugiere el que buena parte de los ejemplos de estos plurales paucales que aparecen en la base de datos ArabiCorpus proceden precisamente de obras gramaticales en las que se trata la cuestión del plural y se aportan ejemplos no necesariamente ligados a la producción de textos reales.

$\mathrm{Si}$ atendemos a los casos en que un singular $f a^{\prime}$ al tiene un plural $a f^{\prime} \bar{a} l$ para el paucal y otro plural $f i^{\prime} \bar{a} l$ o $f u ' \bar{u} l$ para el multitudinal, lo cual correspondería a la situación más general, de acuerdo con los datos

${ }^{17}$ Esto es algo que todavía sucede, si bien de forma bastante residual, en algunos sustantivos de esquema $f a^{\prime} l$, en los que se mantiene hasta hoy en día la diferencia entre el plural paucal y el multitudinal. Es el caso de šahr, que recibe el plural ašhur "de 3 a 10 meses" frente al otro plural šuhūr "más de 10 meses". Otros ejemplos, algo menos evidentes, son alf "mil", pl. ālāf y ulüf, sattr "línea", pl. astur y suṭūr y baḥr pl. abhurur y biḥār.

${ }^{18}$ Sìbawayhi, Kitāb, IV, 591.

${ }^{19}$ Esta sección consta de más de 9 millones de palabras, e incluye el texto completo del Corán, recopilaciones de hadīt, adab, textos de gramática y filosofía, todos ellos pertenecientes a los siglos clásicos del islam. Véase arabycorpus.byu.edu para más detalles. 
de Sībawayhi, registramos en nuestro listado lexicográfico 22 casos de alternancia $a f^{\prime} a \bar{a} l / f i$ ' $\bar{l} l$ ( 16 en el Lisān y 19 en Wehr), frente a 19 casos de alternancia $a f^{\prime} a \bar{l} / f u$ ' $u \bar{l}$ (19 en el Lisān y 15 en Wehr). Sin embargo, no logramos apreciar distinción alguna entre unas y otras formas en función de si hay referencia a un número pequeño o grande. Lo que se observa es que en la mayoría de los casos una de las dos formas es residual y ha caído en desuso, si es que alguna vez fue utilizada, mientras que la otra forma es la de uso predominante, casi exclusivo. Es lo que parece suceder con muchos de estos plurales "raros", que apenas se documentan en la base de datos de Parkinson. A modo de ejemplo, podemos citar los dobletes at $\underline{a} r / u \underline{t} \bar{u} r$ (13.254 casos del primer plural, 0 del segundo), aqlām/qilām (3.821 del primero y 2 del segundo) o anmāt/nimāt (4.367 del primero y 2 del segundo), o anyāb/nuyūb (1.104 el primero y 41 el segundo). Estos casos reflejan la pérdida de uno de los dos plurales, probablemente por economía lingüística, y nótese también que tiende a imponerse el esquema $a f^{\prime} \bar{a} l$ sobre los demás, asunto al que volveremos más adelante. Hay algún caso en que ambos plurales se mantienen más o menos vivos en el uso, como ašğān/šuğ̄un (307 del primero y 961 del segundo), pero son los menos.

A continuación, Sỉbawayhi ofrece una información preciosa de carácter intuitivo: el esquema $f i$ 'a $l$ para el plural de gran número de los singulares $f a$ 'al es más frecuente que el esquema $f u$ ' $u l l$ (wa-l-fi 'àlu fì $h \bar{a} \underline{d} a$ aktar $)^{20}$. Esta es una observación interesante que conviene comprobar. Para ello utilizamos el listado lexicográfico elaborado al efecto, que nos permite corroborar la observación intuitiva del lingüista de Basora. Los datos son los siguientes: en el Lisān al- 'arab aparecen 32 casos de $f i{ }^{\prime} a l$ frente a 28 de $f u ' \bar{u} l$, y en el diccionario de H. Wehr hay 30 casos de $f i^{\prime} \bar{a} l$ frente a 24 de $f u ' \bar{u} l$. Cifras muy similares que ponen de relieve el tino y la finura de la intuición lingüística del genio de Basora. A la vista de que apenas hay sustantivos que utilicen simultáneamente ambas formas de plural ${ }^{21}$, estamos, diríase, ante un caso de

${ }^{20}$ Sībawayhi, Kitāb, IV, 570.

${ }^{21}$ Se trata de las siguientes voces: dakar, que en el Lisān al- 'arab recibe 6 plurales distintos, entre ellos $\underline{d i k} \bar{a} r$ y $\underline{d} u k \bar{u} r, r a \breve{g} a b$, que recibe, entre otros, los plurales arğg $\bar{a} b$, ri $\ddot{g} \bar{a} \bar{b}$ y ruğūb, y našaz, con plurales nušūz, anšăz y nišāa. Tal vez podría añadirse $d \bar{a} r$, con plurales diyār y $d \bar{u} r$ (entre otros). Es notable que el diccionario moderno de Hans Wehr no

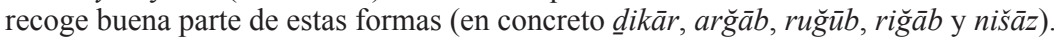


alomorfía prácticamente libre. No parece haber criterio fonético, ni cuantitativo, ni semántico, que determine la selección de una u otra forma de plural.

El siguiente paso que da el prócer de los gramáticos árabes en su descripción de los plurales de fa 'al es señalar que, cuando se supera el número pequeño, en ocasiones se recurre a los esquemas fu 'lān y fi lān. Los ejemplos que aporta para el primer esquema son humlān y sulqān "vegas", y para el segundo hirbān, birqān y wirlān. Y acto seguido afirma que, para el caso de no superarse el número pequeño, su usan los plurales de esquema $a f^{\prime} \bar{a} l$, esto es: $a h m \bar{a} l$, aslāq, $a h r a \bar{a} b, a b r \bar{a} q$ у awrāl.

El análisis de los datos lexicográficos nos indica que fu 'ān y fi ' lān son dos esquemas de uso algo menor (17 veces el primero y 10 el segundo en el listado elaborado a partir del Lisān al- 'arab). Llama la atención que en la gran mayoría de los casos, y en la totalidad de los ejemplos esgrimidos por Sībawayhi, el singular contiene una consonante sonante, sea la líquida [1], la vibrante [r], o las semiconsonantes [w] o [y]. En concreto, de los 17 casos de fi lān solo hay dos que no incluyen [r], [1], [w] o [y]: dihbāan y šibtâan, pero sí incluyen la consonante bilabial sonora [b]. Y de los 10 casos de fu lān solamente tres carecen de ese tipo de consonantes: hudțān, huššān y duhbbān, pero en dos de ellos con [b]. Es decir, que hay un claro condicionante fonético que favorece y determina el uso de estos dos esquemas de plural de número grande, la presencia en el entorno consonántico del singular de una sonante, a la que por proximidad se asimila la bilabial sonora [b]. Parece, por lo tanto, que no es un rasgo meramente léxico, sino que hay una serie de condicionantes fonéticos y morfológicos, una suerte de correspondencia más o menos fija.

Por otro lado, tal y como sucedía con los casos de alternancia $a f^{\prime} \bar{a} l / f i ' a \bar{l}$ y $a f^{\prime} \bar{a} l / f u ' \bar{u} l$, los datos que arrojan las búsquedas efectuadas en las bases de datos no permiten afirmar que haya una diferencia real entre ambas formas ( $a f^{\prime} \bar{a} l v$ s. fu 'lān o $f i$ ' lān) basada en el contraste entre paucal y multitudinal. Lo que se documenta es que, ya desde los primeros siglos del islam, una de las dos formas resulta claramente preponderante sobre la otra, que se convierte en algo residual, sin que se aprecie un uso de una forma para pequeño número y otra para gran número, tal como indica el lingüista de Basora. Por ejemplo, el plural $a b w a \bar{b} b$ se impone con rotundidad al plural bībān, con 19.460 casos do- 
cumentados frente a solo 188 casos en el ArabiCorpus, sin que se aprecie el uso del primero para paucal y el del segundo para multitudinal ${ }^{22}$. Algo similar puede decirse de otros pares de plurales, como a $\dot{g} w \bar{a} r$ (2.097 casos) frente a $\dot{g} \bar{i} r \bar{a} n$ (97), o, en sentido contrario, awrāl (87 casos) frente a wirlān (292 casos). Es decir, la misma conclusión a la que llegábamos antes: una de las dos formas va cayendo en desuso, y la discriminación basada en el número, que es elemento fundamental en la descripción pormenorizada de Sībawayhi, no parece resultar funcional ni operativa.

La siguiente información que ofrece Sībawayhi en su descripción de los plurales fractos del esquema singular fa' $a l$ es sumamente relevante. Dice que en ocasiones se prescinde de los esquemas de multitudinal, y que la esfera semántica que deberían ocupar dichos esquemas queda cubierta también por el esquema más habitual para el paucal, esto es, $a f^{\prime} a \bar{l}$. Como ejemplo de los sustantivos que únicamente reciben esta forma de plural, el sabio de Basora menciona qatab "basto" pl. aqtāb y rasan "cabestro, ronzal" pl. arsān. Y, en efecto, el análisis del listado lexicográfico elaborado a partir del Lisān al- 'arab y del diccionario árabe - inglés de Hans Wehr muestra un elevado número de sustantivos de esquema $\mathrm{fa}$ ' $a l$ para los que solo se documenta una forma de plural: af'äl. Son 145 de un total de 265 en Lisān al$a^{\prime} r a b^{23}$ y 150 de un total de 263 en Hans Wehr. Afinando un poco más la observación, es de notar que hay una serie de sustantivos $f a$ 'al que no tienen un plural af'āl en el Lisān al- 'arab pero sí lo tienen en Hans Wehr, lo cual viene a indicar que para los plurales creados en época posclásica y moderna el esquema $a f^{\prime} \bar{a} l$ funciona como el esquema por defecto, es decir, la primera opción de plural fracto. Los ejemplos do-

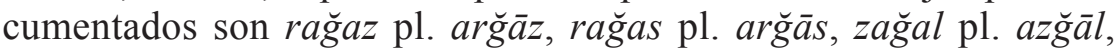
samar pl. asmār, šarak"red" pl. ašrāak, darar pl. aḍrār, 'atab pl. a tāab,

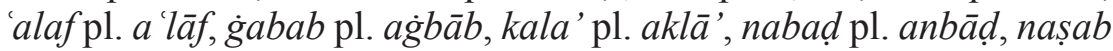
pl. anșāb, nażar pl. anzāar, haram pl. ahrām, y wă̆al pl. awğăl. A esto se añade que en los casos ya mencionados anteriormente en que un

${ }^{22}$ Una serie de búsquedas efectuadas con ese propósito, como las de las cadenas talātatu bībān frente a talātatatu abwāb, o abwābun kațīra frente a bībānun kațira, no arrojan resultados dignos de consideración.

${ }^{23}$ Resulta notable que en este diccionario se señala expresamente que algunos sustantivos no admiten otro esquema de plural que $a f^{\prime} a \bar{l}$. Se trata, por ejemplo, de hanak pl. ahnāk y sanad pl. asnād. 
mașdar admite una forma de plural por haberse desplazado semánticamente de la denotación de la acción en sí misma al producto o la consecuencia de la acción, adquiriendo así un sentido más concreto, el esquema elegido es constantemente $a f^{\prime} \bar{a} l$. Los ejemplos detectados ${ }^{24}$ son: țarab pl. ațrāb, tama' "pl. ațma', 'ăgab pl. a' $\breve{g} \bar{a} b$, 'adam pl. a'dām, farah pl. afrāh, qalaq pl. aqlāq, nazar pl. anżār, hadaf pl. ahdāf y wasah pl. awsāh. De estos datos se desprende que hay una fuerte tendencia a simplificar la alomorfía en los esquemas de plural aptos para el singular $f a^{\prime} a l$, y reducirla a un solo esquema, que se va haciendo más y más dominante: $a f^{\prime} a \bar{l}$, en principio, según Sībawayhi, un plural de pequeño número, pero que adquiere valores nuevos ${ }^{25}$. El propio genio de Basora intuye la situación cuando afirma lo siguiente: "wa-ț-tabātu fì bābi fa 'lin 'alá l-af'āli akțaru min at-tabāti fi bābi fa'lin 'alà l-af'ul"'26. Es decir: "la frecuencia del plural af'āl para un singular $f a$ 'al es mayor que la del plural af'ul para un singular $f a$ ' $l$ '. Parece evidente que esa tendencia en dirección a $a f^{\prime} \bar{a} l$ estaba ya presente en la fase de la lengua árabe que Sībawayhi describe, pero es difícil precisar si hubo algún momento anterior en que la distinción entre plural paucal y multitudinal, al menos para el esquema $f a$ ' $a l$, resultaba productiva y funcional. Probablemente no, y en realidad Sỉbawayhi está describiendo una situación irreal en la que la alomorfía tendría una función de discriminación semántica muy clara ${ }^{27}$. Pero ante la falta

${ }^{24}$ La mayoría de los plurales que se citan a continuación no aparecen registrados en el Lisān al- 'arab, pero sí en los diccionarios modernos como el de Hans Wehr o el de Julio Cortés.

${ }^{25}$ En época contemporánea, se da la curiosa circunstancia de que algunos sustantivos de esquema $f a$ ' $a l$ sustituyen, para acepciones nuevas, el plural esperable vista la tendencia detectada, esto es, af ' $\bar{a} l$, por un plural regular con sufijo - $\bar{a} t$. Es el caso de talab pl. țalabāt en el sentido de "solicitud; pedido" (solamente 3 casos dudosos en el corpus premoderno, 35 casos en el corpus de literatura moderna y 8.733 casos en el corpus de prensa en la base de datos de D. Parkinson) o el de sanad pl. sanadāt, en el sentido financiero de "bono; título" (ningún caso en el corpus premoderno, 1 caso en el corpus de literatura moderna y 4.696 en el corpus de prensa). Este último sustantivo tiene, para el sentido clásico de "fundamento; documento, referencia", un plural $a f^{\prime}$ ' $l$ bien representado en los diccionarios y en el uso. En el Lisān al- 'arab se afirma, incluso, que no hay otra forma de plural que asnād, tal como hemos señalado supra.

${ }^{26}$ Sībawayhi, Kitāb, IV, 572.

${ }^{27}$ En este sentido es plausible pensar que Sībawayhi pudo inspirarse en los métodos de su maestro, al-Halīl ibn Aḥmad al-Farāhīdī, quien hizo una descripción detallada del sistema métrico del árabe clásico (el 'arūọ) en la que, con el fin de que los círculos que ideó resultaran completos, introdujo algunas formas y licencias no documentadas en la 
de información fiable y abundante sobre épocas anteriores, no procede pronunciarse con rotundidad al respecto.

Señala Sỉbawayhi a continuación que en ocasiones un singular esquema $f a^{\prime} l$ puede tomar un plural de esquema $a f^{\prime} u l$ en lugar de $a f^{\prime} a \bar{l}$, para lo cual aduce algunos ejemplos como azmun y ăgbul. En este último caso, el lingüista de Basora parece dudar un tanto de esta forma cuando dice: wa-balaganā anna ba 'dahum yaqūl: 乌̆abal ăgbul "y nos ha llegado la especie de que alguno de ellos [los árabes] dice jabal (sg.) ajbul (pl.)". En el listado léxico que manejamos, se documentan 13 casos de $a f^{\prime} u l$ en el Lisān al- 'arab y solo 5 en Hans Wehr, lo cual evidencia la tendencia a la desaparición de dicho plural. De todas formas, si examinamos de cerca los ejemplos, vemos que se trata de formas desusadas, de muy poca presencia en el uso real de la lengua. De varios de esos plurales no hay vestigios ni en las fuentes premodernas ( $\bar{a} s u d$, $\bar{a} k u m$, abqur, atmun, adwur) y para otros los testimonios recogidos proceden, en su gran mayoría, de tratados lingüísticos clásicos en los que se tratan estas formas poco frecuentes de plural fracto (ăgbul, arḥin, azmun, a 'șin, aqfin, aqwu', anwur y anyub) ${ }^{28}$.

El padre de la gramática árabe indica también que otra posible forma de plural para estos singulares es $f u$ ' $l$, si bien añade que se trata de algo escaso. Los ejemplos aportados son asad pl. usd y watan $\mathrm{pl}$. $w u \underline{t n}$. Señala el lingüista de Basora que estas formas podrían responder a variantes de lectura coránica, y que algunos árabes dicen nașaf (sg.) y $n u s f($ pl.). El examen atento del listado de plurales documentados en Lisān al- 'arab revela unos cuantos ejemplos más: ğuml, 'uğm, 'usl, fulk y wuld, todos ellos de poca raigambre, tanto en árabe de fase antigua como de fase moderna, y todos ellos, salvo quizá el caso de ğuml, con un alomorfo af'āl de uso frecuente. Los datos confirman, por lo tanto, la validez de la información aportada por Sībawayhi.

realidad, pero que le servían para "cuadrar el círculo" y trazar un sistema cuasi perfecto en una aproximación de carácter generativo avant la lettre. Véase al respecto SánchezSancha ("Introducción exegética a la métrica tradicional árabe", pp. 74, 134-138), Paoli (De la théorie à l'usage, pp. 84-94), o Bin 'Abdallah As-Sālim ("Al-Ḥalīl ibn Aḥmad 'arūḍiyyā", pp. 227-229).

${ }_{28}$ Procede señalar, como una muestra más de la vitalidad del esquema af ${ }^{\prime} \bar{a} l$, que sí se registran bastantes casos en los que el plural de pequeño número del esquema $\mathrm{fa}$ ' $l$, que es en principio $a f^{\prime} u l$, es sustituido por $a f^{\prime} \bar{a} l$. Véase Sībawayhi, Kitāb, IV, 568, que indica que este plural $a f^{\prime} \bar{a} l$ para singulares $f a^{\prime} l$ no es propio de la lengua de los árabes, si bien los datos de uso de la lengua apuntan a que es un plural frecuente. 
En el apartado dedicado a los singulares con raíces sordas y defectivas, Sībawayhi indica, al igual que hacía en el apartado dedicado al esquema $f a^{\prime} l$ de raíces regulares, que su comportamiento es similar al de los singulares regulares. Sin embargo, nuevamente nos ofrece el lingüista de Basora datos y observaciones precisos de gran interés, como que el singular rahà solo admite una forma de plural: $\operatorname{arhha} \bar{a}^{\prime}$, lo mismo que sucede con otros dos singulares regulares: qadam pl. aqdām y rasan pl. arsān. Añade a continuación que de existir otros plural sería por analogía (qiyās), pero que él no lo ha oído (lam asmachu). Este dato es muy interesante, porque los diccionarios clásicos incluyen otros plurales de estas dos voces, en contra de lo defendido por Sībawayhi. Se trata, para rahà, de ruḥ̄ (presente en Lisān al- 'arab y en Wehr), arhiya (en ambos diccionarios), arhin ( $a f^{\prime} u l$, solo en Lisān al- 'arab) y un raro rihiyy (solo en Lisān al- 'arab). Esto invita a plantearnos si se trata de derivaciones o variantes posteriores a la época de Sībawayhi o bien son formas que el lingüista de Basora no tiene recogidas por falta de información, o porque las considera poco ortodoxas. Estos casos serían nuevas muestras de cierto "bloqueo" del sistema de distinción entre plural de pequeño número y de gran número, en los que una de las formas, en este caso la de pequeño número, ocupa el lugar de las otras.

El último apartado en la descripción de Sībawayhi está dedicado a los singulares con raíces cóncavas (2w/y). Es muy notable que lo primero que se señala es que nuevamente hay un contraste entre una forma de paucal $\left(a f^{\prime} \bar{a} l\right)$ y una de multitudinal $\left(f i^{\prime} l \bar{a} n\right)$. Los ejemplos que aporta

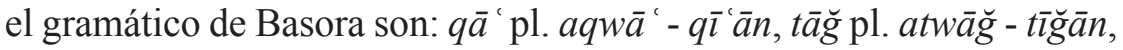
$\breve{g} \bar{a} r$ pl. a $\breve{g} w \bar{a} r-\breve{g} \bar{\imath} r \bar{a} n$. Pero sucede nuevamente que si acudimos al uso real en la lengua de estos plurales, se observa una cierta contradicción entre lo que nos dice Sībawayhi y lo que comprobamos en las bases de datos, como si el genio de Basora estuviera describiendo en realidad un registro o variante lingüística diferente. $Y$ es que en el corpus premoderno de la base de datos de Parkinson solo aparecen 4 casos de $a q w \bar{a} \bar{c}^{\prime}$ (y tres de ellos proceden precisamente de citas de gramáticos, voces testigo en capítulos sobre el plural fracto), frente a 21 de $q \bar{l}^{\prime} \bar{a} n$, 2 de atwāg (ambos recogidos en obras gramaticales) frente a 25 de tı̆ğan, y 7 de ăgwār (2 de ellos de obras gramaticales) frente a 351 de ğìrān.

Otro dato de interés es el que aparece a continuación: los árabes, según Sībawayhi, evitan el uso de fu 'ūl para singulares cóncavos por 
la reluctancia a vocalizar con una $u$ una consonante $/ \mathrm{w} /$ seguida de una vocal larga $\bar{u}$, con lo cual se inclinan claramente por el plural $f i$ ' $\bar{a} n$. Es decir, de nuevo una constricción fonética que provoca la alomorfía en estos esquemas de plural fracto.

\section{Conclusión}

La descripción que hace Sībawayhi de los diversos esquemas de plural fracto del singular $f a$ ' $a l$ es, como el resto del Kitāb, un feliz intento de sistematización de los datos de la lengua de los árabes, apoyado en información real recogida de los beduinos y de las fuentes clásicas, en la fina intuición del genio de Basora. Su forma de enfrentarse a la alomorfía tan abundante y aparentemente anárquica de este apartado de la morfología nominal consiste en presentar los datos de forma ordenada y tratar de buscar una serie de elementos discriminantes que pueden regular la elección de una forma u otra para el plural, creando así unos paradigmas completos y regulares ${ }^{29}$. El discriminante principal ${ }^{30}$ es el de la oposición paucal vs. multitudinal. Sībawayhi presenta una oposición consistente y sistemática entre $a f^{\prime} \bar{a} l$ (forma de pequeño número por excelencia) y el par $f u^{\prime} u \bar{l} l / f i$ 'a $l$ (las dos formas básicas de gran número, en alternancia cuasi libre). Luego hace el estudio de casos más particulares, que son los de formas menos frecuentes, o menos "básicas", como fi 'lān y fu 'lān, cruces y conflictos entre el esquema de pequeño número y los de gran número, y algunas particularidades fonéticas de sustantivos con reduplicación consonántica, cóncavos y defectivos. Pero el propio Sībawayhi reconoce en más de una ocasión que esos elementos discriminantes no funcionan de forma regular, y que con frecuencia el esquema de pequeño número invade la esfera de uso del de gran número. Y no solo Sībawayhi, sino también

${ }^{29}$ Esto nos hace recordar el método seguido por su maestro, al-Halîl ibn Ahmad alFarāhīdī, en su obra lexicográfica $A l$ - 'Ayn, en la que explora las diferentes combinaciones de las consonantes que integran cada una de las raíces árabes, el llamado al-ištiqāq alakbar, incluyendo incluso las que no tienen uso real, al estilo de lo que hizo en su sistematización de la métrica clásica, como señalábamos supra. Véase, por ejemplo, Al-Buqā '̄̄ ("Nazarātu fì mu' '̆gam al- 'ayn", pp. 391-394).

${ }^{30}$ Hemos hecho referencia previamente a otro discriminante menor, el fonético, que opera en algunos casos, como el de los sustantivos cóncavos o el de sustantivos con r/l. 
los hechos documentados de la lengua, apuntan en esa dirección. Cierto es que en las fuentes lexicográficas y gramaticales árabes, como el Lisān al- 'arab, se indica aquí y allí que alguna forma concreta es de uso específico para gran número o para pequeño número ${ }^{31}$, pero se trata de observaciones aisladas que no parecen reflejar un sistema coherente de discriminación numérica entre uno y otro plural. Al menos ese es el resultado que arrojan las búsquedas efectuadas en bases de datos, tal como hemos tratado de poner en claro en este estudio: el esquema de plural $a f^{\prime} \bar{a} l$ no puede, en virtud de su frecuencia y perfiles de uso, considerarse un plural paucal, sino más bien un plural general, válido tanto para pequeño como para gran número. Además, se trata del esquema preponderante, de acuerdo con los datos estadísticos, que muestran a las claras que se trata de la primera opción, la más habitual. Según nuestros datos, el Lisān al- 'arab recoge 188 casos de af'äl frente a 32 de $f u$ ' $\bar{u} l, 29$ de $f i$ ' $a l, 17$ de $f i$ ' län y 10 de $f u$ ' lān, en tanto que Wehr incluye recoge 182 casos de $a f^{\prime} a \bar{l}$ frente a 24 de $f u^{\prime} \bar{u} l, 20$ de $f i^{\prime} \bar{a} l, 13$ de fi 'lān y 5 de $f u$ ' $\bar{a}{ }^{32}$. Es decir, que una descripción basada en los hechos documentados en las fuentes escritas debería comenzar por indicar que a un singular $f a^{\prime} a l$ le corresponde un plural fracto $a f^{\prime} a \bar{l}$ en la mayor parte de los casos, y en menor medida otras formas como $f i{ }^{\prime} \bar{a} l$, fu ' $\bar{u} l$, fi ' lān (especialmente para sustantivos cóncavos y sustantivos con $\mathrm{r} / \mathrm{l}$ ), fu 'lān (especialmente para sustantivos con $\mathrm{r} / \mathrm{l}$ ), amén de algunas otras formas "raras" de escasa prevalencia.

Ahora bien, esto no ha de entenderse como un intento de enmendarle la plana al gran gramático árabe, ni de pretender que su descripción fuerza la realidad y se encamina hacia una sistematización "ficticia", ajena a los hechos reales de la lengua. Y es que las fuentes que manejamos hoy día, las del legado lexicográfico, gramatical y literario de la lengua árabe a lo largo de su historia, no nos ofrecen seguramente la misma información que rumiaba y procesaba, allá por el siglo VIII, Sibawayhi, el genial lingüista de Basora.

${ }^{31}$ Por ejemplo, se dice que ah̆ğàr, adwur y aqfā' son formas de paucal, mientras que $h u$ 'u $l, d \bar{u} r, \mathrm{y}$ qufiyy son formas de multitudinal. Véanse las entradas $\{\mathrm{h} g \mathrm{~g} \mathrm{r}\},\{\mathrm{dwr}\}\{\mathrm{qfy}\}$ $\mathrm{y}\{\mathrm{hyl}\}$.

${ }^{32}$ Véanse los datos de Murtonen (1964), que recoge 79 casos de $a f^{\prime} a \bar{l}$ frente a 18 de

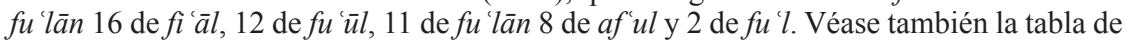
Ratcliffe (The broken plural problem in Arabic and comparative Semitic, p. 75), en la que se documentan 118 casos de $a f^{\prime} \bar{a} l, 15$ de $f u^{\prime} \bar{u} l$ y 11 de $f i^{\prime} \bar{a} l$. 


\section{Referencias}

Baalbaki, Ramzi Munir, "The Book in the grammatical tradition: development in content and method", en Atiyeh, G. N. (ed.), The Book in the Islamic World, Albany, 1995, pp.123-137.

Bin 'Abdallah As-Sālim, Aḥmad, "Al-Halīl ibn Aḥmad 'arūḍiyyā”, en Al- Hal̄̄l ibn Ahmad al-Farāhīd̄̄, Awrāq an-nadwa ad-duwaliyya llatī 'uqidat fì ğāmi 'at Āl al-Bayt 23-25 tammūz 2006, ed. Sa '̄id Ğabr Abū Hुaḍr y Muhammad Maḥmūd ad-Durūbī, Manšūrāt Ǧāmi at Āl al-Bayt, Al-Mafraq, 2007, pp. 223-241.

Al-Būqā̄'̄, Muḥammad, "Naẓarātu fì mu' ğam al- 'ayn, en Al- Halīl ibn Ahmad

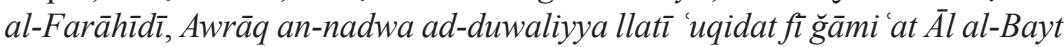
23-25 tammūz 2006, ed. Sa īd Ğabr Abū Haḍr y Muhammad Mahmūd adDurūb̄̄, Manšūrāt Ğāmi 'at Āl al-Bayt, Al-Mafraq, 2007, pp. 391-401.

Corriente, Federico, Problemática de la pluralidad en semítico. El plural fracto, Madrid, CSIC, 1971.

Cortés, Julio, Diccionario de árabe culto moderno, Madrid, Gredos, 1996.

Dayf, Šawqīi, Taysīrāt lugawiyya, El Cairo, Dār al-maª̄rif, 1990.

Nağātī, Aḥmad Yūsuf y an-Nağğāār, Muḥammad 'Alī (ed.), Ma 'ānī l-Qur'ān liabī Zakariyyā al-Farrā', El Cairo, Dār al-Kutub al-Mișriyya, 1980.

Ferrando Frutos, Ignacio, "El plural fracto en semítico. Nuevas perspectivas", Estudios de Dialectología Norteafricana y Andalusí, 4 (1999), pp. 7-23.

Ferrando Frutos, Ignacio, Introducción a la Historia de la lengua árabe. Nuevas perspectivas, Zaragoza, Navarro \& Navarro, 2001a.

Ferrando Frutos, Ignacio, "Sībawayhi y el concepto de jam 'u l-qilla", Al-Qantara, 22/2 (2001), pp. 271-297.

Ferrando Frutos, Ignacio, "The Plural of Paucity in Arabic and Its Actual Scope: On two claims by Siibawayhi and Al-Farraa'", en Boudelaa, Sami (ed.), Perspectives on Arabic Linguistics XVI. Papers from the sixteenth annual symposium on Arabic linguistics, Cambridge, March 2002, Cambridge University, Current Issues in Linguistic Theory 266 xii, 2014, pp. 39-61.

Ferrando Frutos, Ignacio, "Plurals of Paucity in Arabic. From Ancient to Modern Usage", Folia Orientalia, 42/43 (2006-2007), pp. 29-39.

Ferrando Frutos, Ignacio, "Sībawayhi and the broken plural", Jerusalem Studies in Arabic and Islam, 37 (2010a), pp. 53-60.

Ferrando Frutos, Ignacio, "Broken versus regular plural in Modern Arabic: the case for taf 'il", Juan Pedro Monferrer Sala y Nader al-Jallad (ed.), The Arabic language across the Ages, Wiesbaden, Verlag, 2010, pp. 107-117.

Ferrando Frutos, Ignacio [en prensa], "Al-muțābaqa bayna n-na'ti wa-l-man 'ūti fì riwāyāti Yūsuf Zīdan. Namādiğu hāriğgatun 'ani 1-ma āyīri 1-muttaba 'ati fī 1- 'arabiyyati 1-hadīta", en Materials for the Establishment of Contemporary Written Fuṣhā Descriptive Grammars, Aix-en-Provence, pp. 234-253. 
Hārūn, 'Abd as-Salām Muhammad [ed], Kitāb Sībawayhi, Beirut, 'Ālam al-kutub, IV vol, 1983.

Hetzron, Robert, “Two Principles of Genetic Classification”, Lingua, 38 (1976), pp. 89-106.

Ibn Manẓūr, Lisān al-'arab, Beirut, Dār Sādir, XV vol., s.f.

Levin, Aryeh, "Sībawayhi's attitude to the language of the Quran", Israel Oriental Studies, 19 (1999), pp. 267-272.

Lloret, M.R., "La alomorfía en la teoría de la optimidad", Teoría morfológica y morfología del español, Madrid, Ediciones de la Universidad Autónoma de Madrid, 2011, pp. 133-162.

Paoli, Bruno, De la théorie à l'usage. Essai de reconstitution du système de la métrique arabe ancienne, Damasco, Institut Français du Proche-Orient, 2008.

Ratcliffe, Robert, The broken plural problem in Arabic and comparative Semitic, Amsterdam/Philadelphia, John Benjamins, 1998.

as-Sāmarrā'̄̄, Ibrāhīm, Fiqh al-luga al-muqāran, Beirut, Dār al-cilm li-l-malāyīn, 1987.

Sánchez-Sancha, Alcaén, "Introducción exegética a la métrica tradicional árabe”, Awrāq Ğadida, 7-7 (1984-1985), pp. 47-173.

Zaborski, Andrzej, "How Conservative and How Innovating is Arabic?", en Arabic in Context, ed. Ahmad Al-Jallad, Leiden, Brill, 2017, pp. 35-50.

Recibido: $02 / 09 / 2018$

Aceptado: 15/11/2018 